\title{
INDICADORES BIBLIOMÉTRICOS DE LA PRODUCCIÓN CIENTIIFICA PERUANA EN PLANTAS MEDICINALES
}

\author{
Yolanda Angulo-Bazán (101,a \\ Centro Nacional de Salud Intercultural, Instituto Nacional de Salud, Lima, Perú. \\ a Médica cirujana
}

\section{RESUMEN}

Objetivo: Describir la producción científica sobre plantas medicinales con, al menos, un autor con filiación peruana publicada entre el 2000 y el 2019. Materiales y métodos: Se realizó un estudio bibliométrico mediante una búsqueda sistemática en la base de datos Scopus, de donde se seleccionaron artículos originales o comunicaciones breves sobre plantas medicinales que tuvieran, al menos, un autor con una filiación institucional peruana. Se describen las características de las publicaciones, instituciones, países y autores que participaron en estas investigaciones. Se calculó la proporción de colaboración internacional, el índice de transitoriedad, y las redes de colaboración institucional y de coautoria. Resultados: Se incluyeron 200 artículos originales publicados en 83 revistas, con una tendencia creciente, aunque irregular. La institución más productiva fue la Universidad Nacional Mayor de San Marcos, y la revista en la que más se publicó fue Journal of Ethnopharmacology. Las redes de autoría y de colaboración institucional mostraron la influencia de la colaboración internacional en estas publicaciones (53,0\%). Conclusiones: La producción científica peruana sobre plantas medicinales tiene una tendencia creciente, se concentra en ámbitos académicos universitarios y se publica en revistas de alto impacto, debido, en parte, al alto nivel de cooperación internacional existente.

Palabras clave: Ciencia de la Información; Plantas Medicinales; Bibliometría; Descubrimiento del Conocimiento; Edición; Cooperación Internacional; Terapias Complementarias; Artículo de Revista; Comunicación en Salud; Bases de Datos Bibliográficas (Fuente: DeCs BIREME).

\section{BIBLIOMETRIC INDICATORS OF PERUVIAN SCIENTIFIC OUTPUT ON MEDICINAL PLANTS}

\begin{abstract}
Objective: To describe the scientific output on medicinal plants published between 2000 and 2019 with at least one author with Peruvian affiliation. Materials and methods: A bibliometric study was carried out by conducting a systematic search in the Scopus database, from which original articles or short communications on medicinal plants that had at least one author with Peruvian institutional affiliation were selected. The characteristics of the publications, institutions, countries and authors who participated in this research are described. The ratio of international collaboration, the transience index, and the institutional and co-authorship collaboration networks were calculated. Results: A total of 200 original articles published in 83 journals were included. The quantity of articles showed an increasing, though irregular trend during the studied period. The most productive institution was the Universidad Nacional Mayor de San Marcos, and the journal in which most of the articles were published was the Journal of Ethnopharmacology. Authorship and institutional collaboration networks showed the influence of international collaboration in these publications (53.0\%). Conclusions: Peruvian scientific output on medicinal plants shows an increasing trend, observed mainly in academic university settings. The articles are published on high-impact journals, due in part to the high level of international cooperation.
\end{abstract}

Keywords: Information Science; Plants, Medicinal; Bibliometrics; Knowledge Discovery; Publishing; International Cooperation; Complementary Therapies; Journal Article; Health Communication; Databases, Bibliographic (Source: MeSH NLM).

Correspondencia: Yolanda Angulo Bazán; Av. Defensores del Morro 2268, Centro Nacional de Salud Intercultural del Instituto Nacional de Salud, Chorrillos, Lima, Perú; yangulo@ins.gob.pe

\section{INTRODUCCIÓN}

Las plantas medicinales han sido usadas como un recurso terapéutico por los seres humanos desde tiempos ancestrales, y los conocimientos sobre sus propiedades se transmiten de Recibido: $10 / 02 / 2020$ En línea: 21/08/2020 
como insumo para aislar compuestos con actividad biológica necesarios para desarrollar fármacos y tratar diversas enfermedades, estos procedimientos fueron posibles gracias a la aplicación y generación de conocimiento a través del método científico ${ }^{(3)}$.

En los últimos años, el uso de plantas medicinales se ha incrementado en todo el mundo, lo que representa un mercado que ha pasado de sesenta mil millones de dólares en el 2003 a cerca de ochenta mil millones de dólares en el $2012^{(4,5)}$. El Perú es un país con una gran riqueza y diversidad en plantas medicinales. Se considera que su población utiliza, por lo menos, 5000 especies diferentes de plantas en actividades como la alimentación, construcción, artesanías y ornamentos, y por sus propiedades terapéuticas o tóxicas ${ }^{(6)}$. Se tienen evidencias de que su uso se encuentra generalizado en la población, e incluso la práctica de su consumo se realiza mucho antes de acudir a los servicios de salud ${ }^{(7,8)}$.

Pamo-Reyna encontró 45 investigaciones sobre las propiedades de las plantas medicinales publicadas en revistas médicas peruanas en el periodo 2004-2008 ${ }^{(9)}$. No obstante, el contexto de promoción de la investigación científica en nuestro país ha sufrido cambios importantes. Por ejemplo, desde el punto de vista legal se han aplicado principios y medidas, como la Ley que promueve la investigación científica, desarrollo tecnológico e innovación tecnológica (Ley 30309), o la nueva Ley Universitaria (Ley 30220). En ese sentido, hay estudios locales que han dado a conocer el impacto positivo en la producción científica nacional en todos los campos del conocimiento ${ }^{(10)}$, pero se desconoce aún la situación en áreas específicas como el uso de plantas medicinales.

Los estudios bibliométricos contribuyen a determinar el impacto de la producción científica en una determinada área del conocimiento y, mediante el estudio de las redes de colaboración visualizan la dinámica de la producción, analizando autorías, instituciones y países contribuyentes ${ }^{(11)}$. Existen estudios que describen la producción científica sobre plantas medicinales en contextos internacionales ${ }^{(12,13)}$, o el uso de estas en diversas patologías ${ }^{(14)}$; sin embargo, en el Perú existen pocos antecedentes que describan la dinámica de las investigaciones en esta área. Por ello, el objetivo del estudio es describir la producción científica sobre plantas medicinales con, al menos, un autor con filiación peruana, publicada entre el 2000 y el 2019 en revistas indexadas en Scopus.

\section{MATERIALES Y MÉTODOS}

\section{Diseño del estudio}

Se realizó un estudio bibliométrico utilizando la base de datos Scopus. Esta base fue creada en el 2004 y contiene los resúmenes y las citaciones de publicaciones académicas de revistas arbitradas por pares, las cuales pasan por un riguroso proceso de selección, lo que asegura la calidad de la información científica que se brinda ${ }^{(15)}$.

\section{MENSAJES CLAVE}

Motivación para realizar el estudio: Existe poca información respecto del estado actual de la producción científica sobre plantas medicinales en la que participan autores e instituciones peruanas.

Principales hallazgos: Se encontró que la producción científica peruana sobre plantas medicinales tiene una tendencia creciente, además, más de la mitad de los artículos tuvieron participación de autores e instituciones del extranjero, lo que condiciona un mayor impacto, reflejado en el número de citas.

Implicancias: Este análisis reposiciona la producción científica sobre plantas medicinales como una prioridad de investigación a fin de obtener oportunidades de financiamiento.

\section{Población y muestra}

Se realizó una búsqueda sistemática de información en Scopus. Se incluyeron artículos originales o comunicaciones breves (o denominaciones similares) que tuvieran al menos un autor con una filiación institucional peruana (independientemente de la posición del autor), publicados entre el 1 de enero del 2000 y el 31 de diciembre del 2019, en los que se investiguen las propiedades de las especies vegetales reconocidas como «medicinales» en documentos de gestión de los sistemas de salud del Perú. Para ello, se consideró el Catálogo florístico de plantas medicinales peruanas, elaborado por el Instituto Nacional de Salud y el Petitorio nacional de productos, recursos e insumos terapéuticos afines de uso en medicina complementaria del Seguro Social de Salud (EsSalud). Estos documentos ayudaron a la elaboración de un listado de plantas medicinales peruanas (Material suplementario 1), debido a las dificultades existentes en la búsqueda de información sobre plantas medicinales ${ }^{(16)}$.

Finalmente se eligieron los términos de búsqueda: "Plant extracts" OR "medicinal plants" OR "plants, medicinal" $O R$ "phytotherapy OR "medicinal herbs" OR "phytomedicine" (Material suplementario 2).

Se incluyeron estudios cuyos diseños estaban de acuerdo con el proceso de desarrollo de drogas vegetales con perspectiva tradicional ${ }^{(17)}$, ya sea en la fase de descubrimiento (estudios etnobotánicos, observacionales y fitoquímicos) o de desarrollo (estudios preclínicos [in vivo e in vitro] y ensayos clínicos). Se excluyeron estudios en los que se utilizaron sustancias sintéticas basadas en componentes de especies vegetales, estudios sobre propiedades físicas o aspectos de cultivo, revisiones narrativas, revisiones sistemáticas, capítulos de libros, guías de práctica clínica o reportes de caso.

\section{Variables de estudio}

De los artículos seleccionados se recolectó información sobre el año de publicación, el idioma de publicación (inglés, español o portugués), las áreas de conocimiento según la cla- 
sificación de Scopus (farmacología, medicina, bioquímica/ biología molecular, química, inmunología y microbiología, profesiones de la salud, ciencias sociales, entre otros), las instituciones peruanas que participaron en la investigación (frecuencia, índice $\mathrm{H}$, promedio de citas por documento), así como diseños de investigación (estudios etnobotánicos, observacionales, fitoquímicos, preclínicos y clínicos). La clasificación de los artículos por diseños, se realizó de forma manual. Adicionalmente, se describieron las características de las instituciones más productivas (más de cinco artículos publicados), la cantidad de citas acumuladas y las citas por publicación.

Posteriormente, se evaluaron las redes de colaboración institucional y algunos indicadores bibliométricos asociados: porcentaje de colaboración internacional, proporción de autores peruanos en publicaciones colaborativas, países de colaboración frecuente, y la asociación entre el número de citas y la presencia de colaboración internacional.

Asimismo, se determinó el número de autores de estos estudios y se presentaron las características de los autores más productivos (con más de 10 publicaciones), sus filiaciones e índices H. Como indicador bibliométrico se calculó el índice de transitoriedad, definido como la proporción de autores que publicaron solo un artículo en el periodo de estudio.

Finalmente, se identificaron las revistas en las que fueron publicados estos estudios, su país, índice $\mathrm{H}$, cuartil de Scopus, citas por documento y porcentaje de cooperación internacional. Esta información se obtuvo de la página web de Scimago Journal \& Country Rank (https://www.scimagojr.com/).

\section{Construcción de la base de datos}

Los resultados de la búsqueda sistemática se sometieron a un proceso de control de calidad, donde se verificó que los registros cumplieran los criterios de selección. Posteriormente, se guardaron en una lista de Scopus y exportados a archivos de extensión .csv y .ris.

El archivo .ris se exportó al gestor de referencias bibliográficas Mendeley Desktop versión 1.19.4 ® (2008-2019 Mendeley Ltd.), donde se uniformizó la escritura de los autores y se verificaron las posibles duplicaciones. Luego, se analizó en el programa BibExcel $\AA$ versión 2016-02-20, mediante el cual se crearon archivos de extensión .net y .vec, necesarios para elaborar los gráficos de las redes de colaboración. Finalmente, estos gráficos fueron elaborados con el programa Gephi 0.9.2. ® (Gephi contributors, 2008-2017).

\section{Análisis estadístico}

El archivo en formato .xlsl fue importado al programa STATA v.13.0 (College Station, TX: StataCorp LP. 2013 ()) y se obtuvieron frecuencias absolutas, relativas (porcentajes) y medidas de resumen (media y desviación estándar o mediana y rango intercuartilar). La asociación entre el número de citas y la presencia de cooperación internacional se evaluó con la prueba de U de Mann-Whitney, y se consideró estadísticamente significativo un valor de $\mathrm{p}<0,05$. Las figuras y tablas se elaboraron con el programa Microsoft Excel $₫$ 2019.

\section{Aspectos éticos}

Se accedió a Scopus con una licencia institucional (Consejo Nacional de Ciencia y Tecnología) y no fue necesario acceder a las versiones completas de los artículos incluidos en este análisis. Se siguieron las consideraciones éticas de las bases de datos de salud y biobancos de la Asociación Médica Mundial ${ }^{(18)}$.

\section{RESULTADOS}

\section{Indicadores de producción}

La estrategia de búsqueda recuperó 300 artículos, de los cuales se incluyeron 200. Algunas de las causas de exclusión: el objetivo de 35 estudios no era evaluar las propiedades de las plantas medicinales; en 31 artículos evaluaban las propiedades fisicoquímicas y otras relacionadas al cultivo de las plantas; 10 artículos no tenían filiación peruana; y en 10 artículos se utilizaron derivados sintéticos. Adicionalmente, se hallaron dos capítulos de libro, dos revisiones sistemáticas, siete revisiones narrativas, una guía de práctica clínica y dos reportes de caso.

Dentro de las áreas del conocimiento, la mayoría de las publicaciones abarcó temas de farmacología y toxicología $(27,0 \%)$, medicina $(22,7 \%)$, ciencias biológicas/agricultura $(17,4 \%)$, y bioquímica $(13,4 \%)$. Los estudios preclínicos in vivo (en animales) representaron el $29,5 \%$ de los diseños encontrados, seguidos de los estudios preclínicos in vitro $(25,5 \%)$. Adicionalmente, 48 estudios $(24,0 \%)$ tuvieron como objetivo detectar compuestos fitoquímicos y 15 publicaciones $(7,5 \%)$ fueron producto de estudios observacionales transversales que evaluaron el uso de plantas medicinales en distintas poblaciones. Once publicaciones correspondieron a estudios etnobotánicos $(5,5 \%)$ y solo se encontró un estudio con un diseño cualitativo. Catorce estudios clínicos se realizaron en seres humanos, nueve fueron estudios pre-pos $(4,5 \%)$ y cinco fueron ensayos clínicos aleatorizados (2,5\%).

Las investigaciones incluidas fueron publicadas en 83 revistas. Se observó una tendencia polinómica de sexto orden en las publicaciones entre el 2000 y el $2019\left(R^{2}=0,706\right)$, con una tasa de crecimiento anual promedio de $18,6 \%$. Esta tasa fue negativa en los años 2003, 2005, 2008, 2010, 2012, 2014 y 2018 (Figura 1). La mayoría de las publicaciones $(73,5 \%)$ se redactaron en inglés, mientras que solo $51(25,5 \%)$ fueron en español y $2(0,1 \%)$ en portugués.

El artículo más citado fue «Antimicrobial activity of selected Peruvian medicinal plants» de Rojas et al., publicado en 2003 en Journal of Ethnopharmacology, con 179 citas. Esta revista fue la que tuvo más artículos publicados (15,5\%), seguida por la Revista Peruana de Biología (6,5\%) y la Revista 


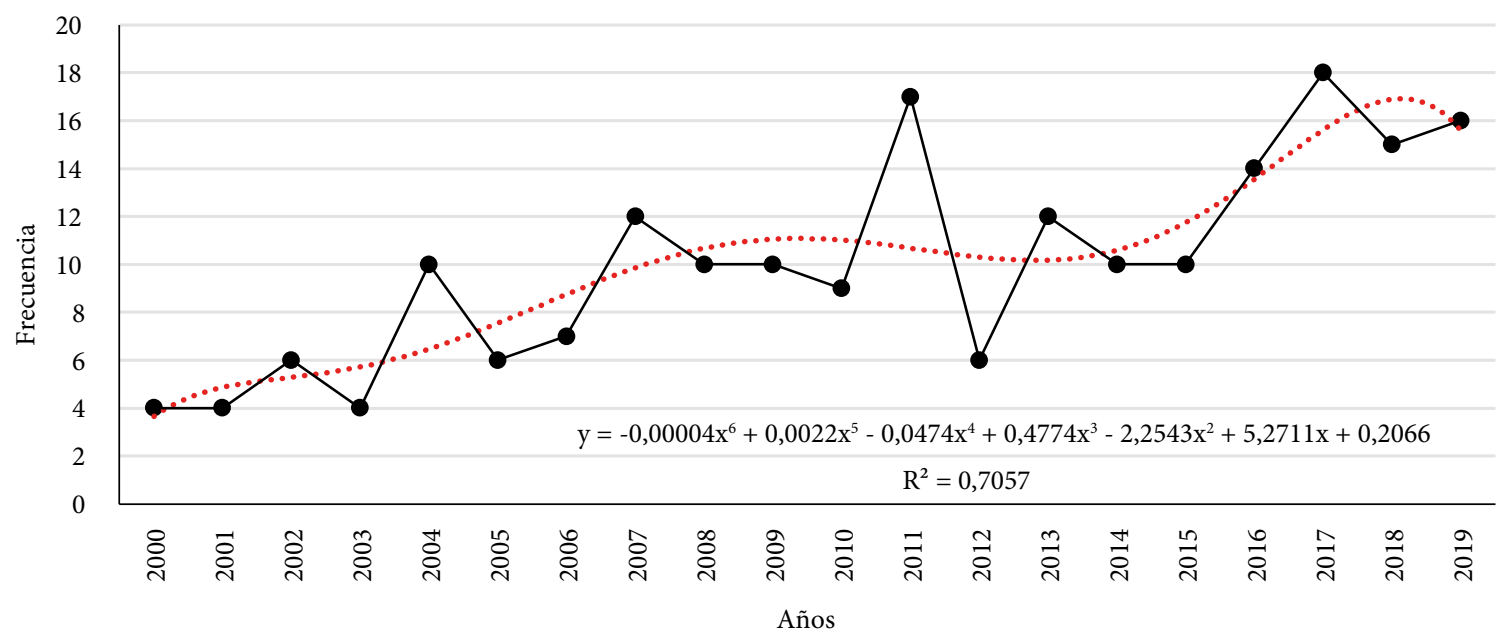

Figura 1. Tendencia de publicación de artículos originales / originales breves sobre plantas medicinales por autores con, al menos, una filiación peruana (2000-2019)

Peruana de Medicina Experimental y Salud Pública (6,0\%) (Tabla 1).

\section{Indicadores de colaboración}

Se encontraron 237 instituciones en las filiaciones de los artículos recuperados, 79 de ellas eran peruanas (33,3\%). La Universidad Nacional Mayor de San Marcos (UNMSM) fue la institución con mayor producción, con 54 artículos, seguida por la Universidad Peruana Cayetano Heredia (UPCH) con 52 artículos y la Universidad Agraria La Molina (UNALM) con 17 artículos; aunque estas dos últimas instituciones tuvieron un promedio de citas por publicación más alto que la UNMSM (Tabla 2).

En el 53,0\% de los artículos se encontró cooperación internacional con 29 países, entre los que destacan Estados Unidos (26 publicaciones), Francia (19 publicaciones) y España (15 publicaciones). En los artículos colaborativos, el porcentaje promedio de la participación de autores peruanos fue de 87,5\% (7,1\%-87,5\%). En 28 (26,4\%) artículos colaborativos el primer autor tuvo filiación peruana y en $19(17,9 \%)$ fue autor corresponsal. Los artículos que tuvieron cooperación internacional tuvieron una mediana de 17 (RIC: 33) citas, mientras que aquellos con solo autores peruanos fueron citados 5 (RIC: 15) veces, encontrándose diferencias significativas $(\mathrm{p}<0,001)$.

Se encontraron 809 autores, de los cuales solo 8 tuvieron una producción mayor o igual a 10 publicaciones. Dentro de los más productivos están Gustavo Francisco Gonzales

Tabla 1. Diez revistas que más frecuentemente publicaron artículos sobre plantas medicinales con, al menos, una filiación peruana (2000-2019)

\begin{tabular}{|c|c|c|c|c|c|c|c|}
\hline Revista & País & $\mathbf{n}$ & $\%$ & Índice $\mathrm{H}$ & $\begin{array}{c}\text { Cuartil } \\
(2018)\end{array}$ & $\begin{array}{l}\text { Citas por } \\
\text { documento }\end{array}$ & $\begin{array}{l}\text { Colaboración } \\
\text { internacional }\end{array}$ \\
\hline Journal of Ethnopharmacology & Países Bajos & 31 & 15,5 & 170 & Q1 & 3,5 & 28,5 \\
\hline Revista Peruana de Biología & Perú & 13 & 6,5 & 18 & Q3 & 0,3 & 21,9 \\
\hline $\begin{array}{l}\text { Revista Peruana de Medicina } \\
\text { Experimental y Salud Pública }\end{array}$ & Perú & 12 & 6,0 & 16 & Q3 & 0,6 & 14,6 \\
\hline Journal of Natural Products & Estados Unidos & 7 & 3,5 & 124 & Q1 & 4,4 & 50,0 \\
\hline Food Chemistry & Países Bajos & 7 & 3,5 & 221 & Q1 & 5,7 & 27,3 \\
\hline $\begin{array}{l}\text { Boletín Latinoamericano y del Caribe de } \\
\text { Plantas Medicinales y Aromáticas }\end{array}$ & Cuba & 6 & 3,0 & 16 & Q3 & 0,5 & 14,3 \\
\hline Andrología & Reino Unido & 6 & 3,0 & 52 & Q2 & 1,8 & 18,2 \\
\hline Revista de Gastroenterología del Perú & Perú & 6 & 3,0 & 14 & Q4 & 0,3 & 34,7 \\
\hline Planta Medica & Alemania & 4 & 2,0 & 103 & Q1 & 2,6 & 31,8 \\
\hline Toxicology Mechanisms and Methods & Reino Unido & 4 & 2,0 & 35 & Q2 & 2,8 & 23,7 \\
\hline
\end{tabular}

Fuente: Scimago SJR 
Tabla 2. Características de las instituciones peruanas con mayor producción científica en investigación sobre plantas medicinales (2009-2019)

\begin{tabular}{|c|c|c|c|c|}
\hline Institución & $\begin{array}{c}\text { Número de } \\
\text { publicaciones }\end{array}$ & $\begin{array}{l}\text { Número de } \\
\text { autores }\end{array}$ & Número de citas & $\begin{array}{c}\text { Citas por } \\
\text { publicación }\end{array}$ \\
\hline Universidad Nacional Mayor de San Marcos & 54 & 94 & 737 & 13,6 \\
\hline Universidad Peruana Cayetano Heredia & 52 & 80 & 1701 & 32,7 \\
\hline Universidad Nacional Agraria La Molina & 17 & 24 & 531 & 31,2 \\
\hline Universidad Nacional San Luis Gonzaga de Ica & 12 & 5 & 110 & 9,2 \\
\hline Universidad Nacional de la Amazonía Peruana & 12 & 15 & 198 & 16,2 \\
\hline Universidad Nacional de Trujillo & 11 & 20 & 37 & 3,4 \\
\hline Pontificia Universidad Católica del Perú & 10 & 10 & 378 & 37,8 \\
\hline Universidad Nacional Agraria de la Selva & 6 & 3 & 234 & 39,0 \\
\hline Instituto de Investigaciones de la Amazonía Peruana & 5 & 4 & 178 & 29,7 \\
\hline Universidad Nacional de Ingeniería & 5 & 5 & 15 & NA \\
\hline Universidad Nacional de San Agustín de Arequipa & 5 & 11 & 20 & 4,0 \\
\hline Universidad Peruana de Ciencias Aplicadas & 5 & 9 & 39 & 7,8 \\
\hline Instituto Nacional de Medicina Tradicional & 5 & 2 & 174 & 34,8 \\
\hline
\end{tabular}

Fuente: Scopus

con 24 publicaciones (índice $\mathrm{H}$ de 36), Manuel Gasco con 13 publicaciones (índice $\mathrm{H}$ de 21) y Rosario Rojas Durán con 13 publicaciones (índice $\mathrm{H}$ de 16). Cuatro autores tuvieron como filiación a la UPCH, dos tuvieron filiación francesa y dos pertenecían a la UNMSM (Tabla 3). Entre los investigadores, 632 fueron autores de un solo un artículo, lo que brindó un índice de transitoriedad de 78,0\%.

\section{Redes de colaboración}

$\mathrm{Al}$ evaluar los niveles de colaboración interinstitucional se encontró una red de colaboración liderada por la UNMSM y la $\mathrm{UPCH}$, respaldada por instituciones francesas. Asimismo, ambas instituciones colaboran con otras redes de menor magnitud lideradas por la Universidad Nacional de la Amazonía Peruana (UNAP), la Universidad Nacional San Luis Gonzaga y la Pontificia Universidad Católica del Perú (Figura 2).
Al evaluar las redes de colaboración entre autores, se encontró una estrecha colaboración entre tres redes lideradas por Rosario Rojas Durán, Michel Sauvain y Gustavo Francisco Gonzáles; todos investigadores con filiación de la UPCH. Por otro lado, se observa una red dirigida por Oscar Herrera Calderón y Jorge Arroyo Acevedo, que pertenece a investigadores con filiación de la UNMSM. Se observan hasta diez redes de colaboración aisladas, con grupos de investigadores que han publicado por lo menos tres artículos sobre el tema de estudio (Figura 3).

\section{DISCUSIÓN}

La producción científica en plantas medicinales de autores con, al menos, una filiación peruana ha mostrado una tendencia creciente, aunque irregular. Esta tendencia no es ajena a la encontrada en otros estudios; por ejemplo, Wai Kan Yeung et al. realizaron un análisis de las publicaciones

Tabla 3. Diez autores con mayor número de artículos originales con, al menos, una filiación peruana sobre plantas medicinales

\begin{tabular}{lcccc}
\hline Autor & Artículos & Filiación & País & Índice H \\
\hline Gustavo Francisco González & 23 & Universidad Peruana Cayetano Heredia & Perú & 36 \\
Manuel Gasco & 13 & Universidad Peruana Cayetano Heredia & Perú & 21 \\
Rosario Rojas Durán & 13 & Universidad Peruana Cayetano Heredia & Perú & 16 \\
Genieve Bourdy & 12 & IRD Institut de Recherche pour le Developpement & Francia & 12 \\
Jorge Luis Arroyo-Acevedo & 12 & Universidad Nacional Mayor de San Marcos & Perú & 6 \\
Abraham Vaisberg & 10 & Universidad Peruana Cayetano Heredia & Perú & 22 \\
Michel Sauvain & 10 & IRD Institut de Recherche pour le Developpement & Francia & 26 \\
Oscar Herrera Calderón & 10 & Universidad Nacional Mayor de San Marcos / & Perú & 7 \\
\hline
\end{tabular}

Fuente: Scopus 


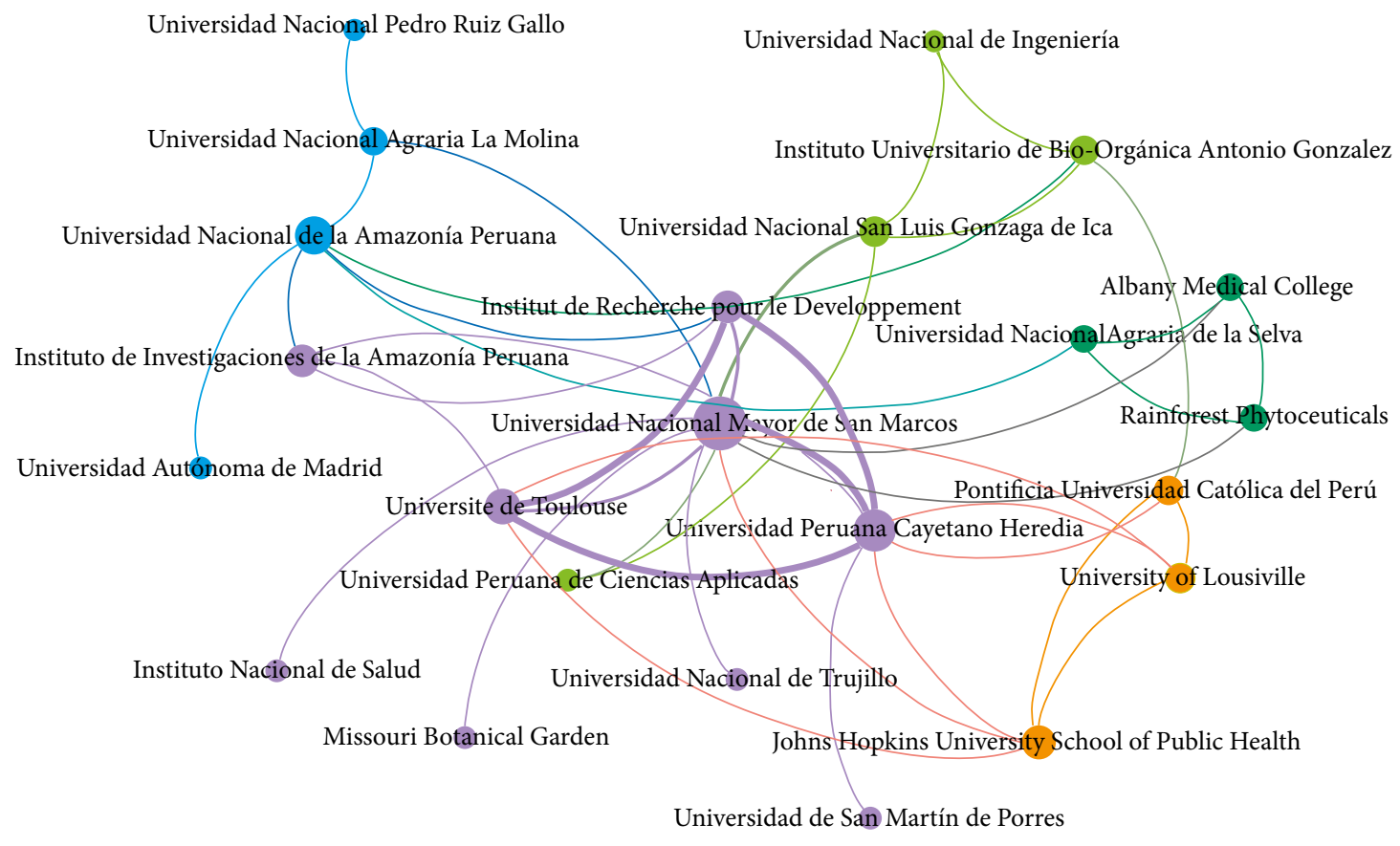

Instituto Nacional de Medicina Tradicional

Universityof Illinois

Figura 2. Redes de colaboración interinstitucional en publicaciones científicas sobre plantas medicinales con, al menos, una filiación peruana (2000-2019). El tamaño del nodo es proporcional a la frecuencia de publicaciones, y el grosor de la arista, a la intensidad de la colaboración. Fuente: Gephi ${ }^{\circledR}$

ligadas a la etnofarmacología indexadas en World of Science de 1958 al 2018, en el cual mostraron una tendencia creciente de publicaciones desde el $2006^{(13)}$. Sin embargo, los resultados no están acorde con estudios de otros países como Cuba, en los que se observa una producción constante desde el $2004^{(19)}$.

Las instituciones involucradas en las publicaciones de estos artículos suelen ser académicas, lo cual muestra la gran importancia que tienen las universidades como entidades generadoras de conocimientos en el uso de plantas medicinales. Estos datos refuerzan los hallazgos realizados por Córdova Rengifo, quien plantea un modelo de interés por la investigación de las plantas medicinales en universidades, nacido de la observación de su uso en la población general, con la motivación de validar desde el punto de vista científico las propiedades medicinales que se les atribuye ${ }^{(20)}$. Asimismo, el documento de consenso de un grupo de expertos en plantas medicinales, promovido por la Organización Panamericana de la Salud menciona que, si bien existe una amplia variedad de líneas de investigación en las universidades, los limitantes en la investigación científica a este nivel se resumen en la falta de laboratorios acreditados para realizar estudios especializados y en la poca cultura de innovación que no incentiva la generación de patentes nacionales ${ }^{(21)}$.

Estas deficiencias pueden explicar parcialmente la alta proporción de colaboración internacional encontrada y las consiguientes redes de colaboración interinstitucionales, lideradas por la UNMSM, UPCH y la UNAP, y respaldadas por instituciones francesas y americanas. Ante esto se debe recordar que la Organización Mundial de la Salud incentiva la formación de redes de investigación en plantas medicinales ${ }^{(22)}$, mientras que los sistemas médicos tradicionales más desarrollados, como la medicina tradicional china o el AYUSH (Ayurveda, Yoga, Unani, Siddha y Homeopatia) ven en la colaboración internacional, un método eficaz para difundir y usar sus recursos naturales en otras poblaciones ${ }^{(23)}$.

$\mathrm{Si}$ bien se observa que la mayor producción de artículos proviene de universidades públicas peruanas como la UNMSM o la UNALM, la mayor cifra de citas por publicación se encontró en los artículos producidos por los investigadores de la UPCH. Asimismo, de forma coherente con la alta proporción de cooperación internacional, se vio que estos artículos fueron más citados en comparación con aquellos que solo incluyeron autores peruanos. Estas características, asociadas a un índice de transitoriedad mayor del $60 \%$ son coherentes con un campo de conocimiento en el que la información científica aún no se encuentra totalmente consolidada ${ }^{(24)}$. Finalmente, la interpretación evidente para los investigadores en estas áreas es que la cooperación internacional les traerá publicaciones de mayor impacto. 


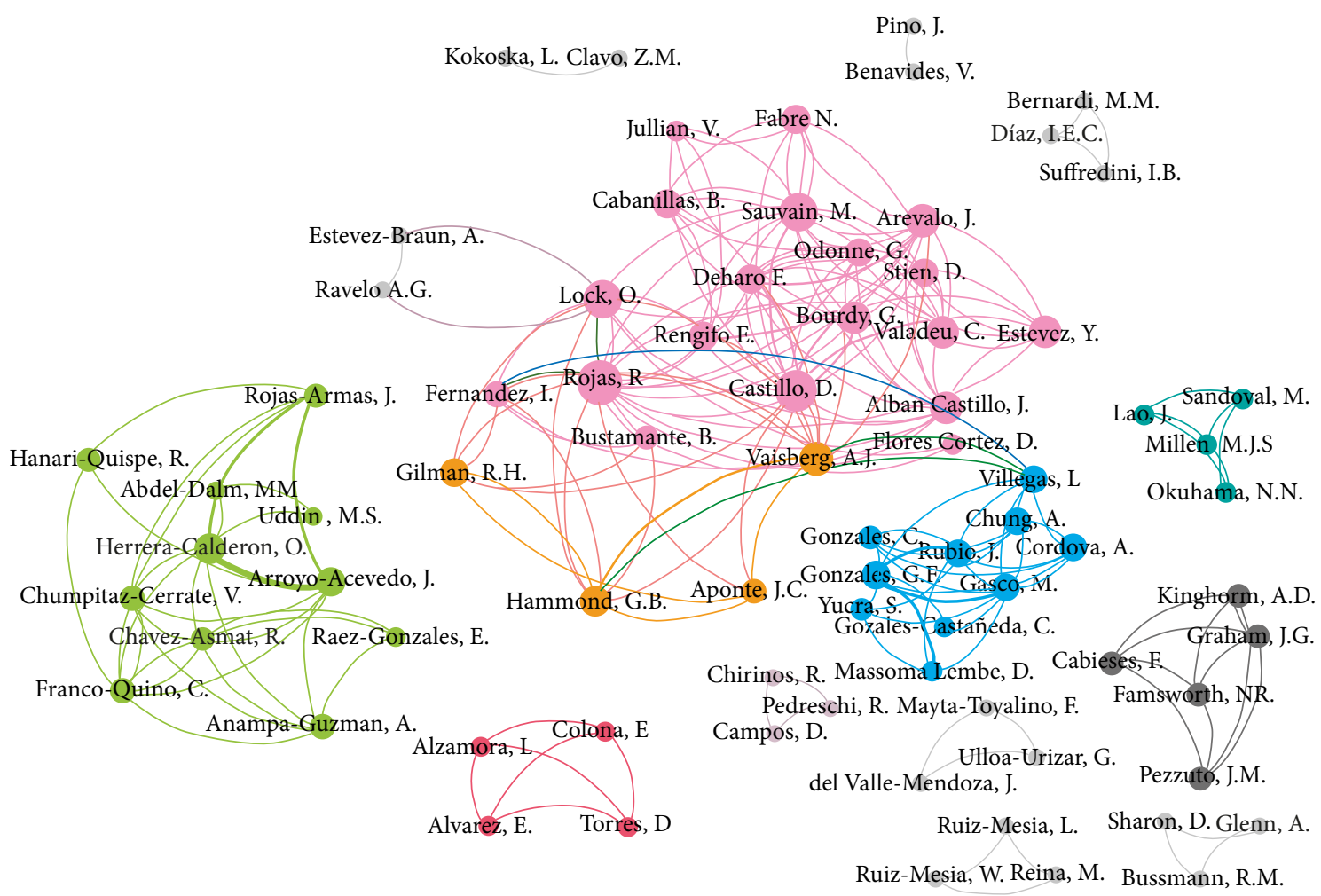

Figura 3. Redes de autoría en publicaciones científicas sobre plantas medicinales con, al menos, una filiación peruana (2000-2019). El tamaño del nodo es proporcional a la frecuencia de publicaciones, y el grosor de la arista, a la intensidad de la colaboración. Fuente: Gephi ${ }^{\circledR}$

Adicionalmente, se destaca que dentro de las revistas con mayor producción de artículos se encuentre una buena proporción de publicaciones que se encuentran en el cuartil 1 (Q1) de Scopus, con The Journal of Ethnopharmacology, como el medio de difusión más utilizado por los investigadores del área. Esta revista es una de las pocas especializadas en la publicación de investigaciones interdisciplinarias en etnofarmacología, etnobotánica y etnoquímica con el objetivo de documentar y preservar este conocimiento ${ }^{(25)}$. Seguidamente, las revistas preferidas por los investigadores para publicar sus hallazgos fueron la Revista Peruana de Biología de la UNMSM y la Revista Peruana de Medicina Experimental y Salud Pública del Instituto Nacional de Salud.

Esta situación refleja algunas de las circunstancias habituales en los grupos de investigación de América Latina, quienes buscan enviar sus manuscritos a las revistas mejor posicionadas, pero que a la vez ofrezcan opciones de acceso abierto y sin costos de publicación ${ }^{(26,27)}$. Sin embargo, estas dificultades no mellan la calidad de los estudios producidos por los investigadores latinoamericanos y, en este caso, por instituciones peruanas; ya que muchos de ellos han sido publicados en revistas Q1 que ofrecen costos de publicación accesibles o a los que se pueden acceder gracias a la cooperación internacional ${ }^{(28)}$.
Dentro de los antecedentes más resaltantes, encontramos que Gonzales Alonso et al. publicaron un estudio donde se buscaba determinar el impacto de las investigaciones científicas sobre plantas medicinales en Scopus, publicadas

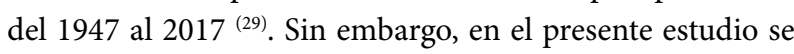
realiza una estrategia de búsqueda mucho más detallada y se obtienen indicadores bibliométricos más exactos, de mayor utilidad para la toma de decisiones. A pesar de ello, ambos estudios coinciden en la tendencia creciente de publicaciones en los últimos años y la importancia de la cooperación internacional en los estudios que llegan finalmente a ser publicados en revistas indexadas.

Por otro lado, una de las limitaciones más grandes de este estudio es que solo expresa lo publicado en una base de datos de relevancia para el ámbito local, pero que no engloba la totalidad de la producción científica del Perú. Por ejemplo, existen 12 títulos de revistas en ciencias de la salud indexadas en SciELO Perú, de las cuales solo tres se incluyen en esta búsqueda por estar también indexadas en Scopus. Asimismo, la búsqueda de información no incluye repositorios académicos, por lo que no se está considerando la producción de tesis de pregrado ni posgrado, lo que podría tener un impacto significativo en esta área del conocimiento, si se considera que este estudio ya evidencia que las universidades son los núcleos 
productivos más importantes de conocimiento en plantas medicinales peruanas.

Otros estudios han resaltado que una de las dificultades más relevantes para un investigador de plantas medicinales es la dispersión de la información necesaria para estructurar un estudio coherente y acorde con el estado del arte actual. Sin embargo, el Perú no cuenta con un repositorio especializado en clasificar y conservar el conocimiento científico generado sobre plantas medicinales, a pesar de ser un recurso natural protegido por la nación.

Se concluye que la investigación científica sobre plantas medicinales con, al menos, un autor con filiación peruana publicada entre el 2000 al 2019 en revistas indexadas en Sco-

\section{REFERENCIAS BIBLIOGRÁFICAS}

1. Mathez-Stiefel S-L, Vandebroek I. Distribution and Transmission of Medicinal Plant Knowledge in the Andean Highlands: A Case Study from Peru and Bolivia. Evid Based Complement Alternat Med. 2012;2012:959285. doi: 10.1155/2012/959285.

2. Caballero-Serrano V, McLaren B, Carrasco JC, Alday JG, Fiallos L, Amigo J, et al. Traditional ecological knowledge and medicinal plant diversity in Ecuadorian Amazon home gardens. Global Ecology and Conservation. 2019;17:e00524. doi: 10.1016/j.gecco.2019.e00524.

3. Singh R. Medicinal Plants: A Review. Journal of Plant Sciences. 2015;3(1):50. doi: 10.11648/j.jps.s.2015030101.18.

4. Allkin B. Useful Plants - Medicines: At Least 28,187 Plant Species are Currently Recorded as Being of Medicinal Use. Dans: Willis KJ, directeur. State of the World's Plants 2017. [Internet]. London (UK): Royal Botanic Gardens, Kew; 2017 [citado el 11 diciembre del 2019]. (Wellcome Trust-Funded Monographs and Book Chapters). Disponible en: www.ncbi.nlm.nih.gov/books/NBK464488/.

5. Akinyemi O, Oyewole S, Jimoh K. Medicinal plants and sustainable human health: a review. Horticult Int J. 2018;2(4):194-195. doi: 10.15406/hij.2018.02.00051.

6. Brack Egg A. Diccionario enciclopédico de plantas útiles del Perú. Cuzco: Programa de las Naciones Unidas para el Desarrollo; Centro de Estudios Regionales Andinos Bartolomé de Las Casas; 1999.

7. Mejía Gálvez JAM, Carrasco E, Miguel JL, Flores SA. Conocimiento, aceptación y uso de medicina tradicional peruana y de medicina alternativa/complementaria en usuarios de consulta externa en Lima Metropolitana. Rev Per Med Integrativa. 2017;2(1):47-57. doi: 10.26722/rpmi.2017.21.44.

8. Oblitas G, Hernández-Córdova G, Chiclla A, Antich-Barrientos M, Ccorihuamán-Cusitito L, Romaní F. Empleo de plantas medicinales en usuarios de dos hospitales referenciales del Cusco, Perú. Rev Peru Med Exp Salud Publica. 2013;30(1):64-8. doi: 10.1590/S172646342013000100013

9. Pamo-Reyna OG. Características de los trabajos publicados sobre las propiedades de las plantas en revistas médicas peruanas. Rev Peru Med Exp Salud Publica. 2009;26(3):314-23. Disponible en: http:// www.scielo.org.pe/scielo.php?pid=s1726-46342009000300008\&scrip$\mathrm{t}=\mathrm{sci} \_$arttext.

10. Consejo Nacional de Ciencia, Tecnología e Innovación Tecnológica. Informe $\mathrm{N}^{\circ} 1$ - Principales Indicadores Bibliométricos de la Actividad Científica Peruana 2006-2011 [Internet]. Lima: CONCYTEC; 2014 Disponible en: http://bvcyt.concytec.gob.pe/images/publicaciones/ principales_indicadores_2006_2011.pdf.

11. Cooper ID. Bibliometrics basics. J Med Libr Assoc. 2015;103(4):217-8 doi: 10.3163/1536-5050.103.4.013. pus, tiene una tendencia creciente, se concentra en ámbitos académicos universitarios y se publica en revistas de diversos cuartiles, incluyendo el Q1, debido, en parte, al alto nivel de cooperación internacional existente.

Contribuciones de los autores: YAB es responsable de la concepción, diseño, análisis e interpretación de los datos del manuscrito, de la redacción y revisión de la versión final del manuscrito y de garantizar las cuestiones relativas a la exactitud o integridad de cualquier parte del estudio.

Financiamiento: Autofinanciado.

Conflictos de interés: La autora declara no tener conflictos de interés.

Material suplementario: Disponible en la versión electrónica de la RPMESP.
12. Salmerón-Manzano E, Garrido-Cardenas JA, Manzano-Agugliaro F. Worldwide Research Trends on Medicinal Plants. Int J Environ Res Public Health. 2020;17(10):3376. doi: 10.3390/ijerph17103376.

13. Yeung AWK, Heinrich M, Atanasov AG. Ethnopharmacology-A Bibliometric Analysis of a Field of Research Meandering Between Medicine and Food Science?. Front Pharmacol. 2018;9:215. doi: 10.3389/fphar.2018.00215.

14. García-García P, López-Muñoz F, Rubio G, Martín-Agueda B, Alamo C. Phytotherapy and psychiatry: bibliometric study of the scientific literature from the last 20 years. Phytomedicine. 2008;15(8):566-76. doi: 10.1016/j.phymed.2008.04.014.

15. Baas J, Schotten M, Plume A, Côté G, Karimi R. Scopus as a curated, high-quality bibliometric data source for academic research in quantitative science studies. Quantitative Science Studies. MIT Press. 2020;1(1):377-86. doi: 10.1162/qss_a_00019.

16. Ningthoujam SS, Talukdar AD, Potsangbam KS, Choudhury MD. Challenges in developing medicinal plant databases for sharing ethnopharmacological knowledge. J Ethnopharmacol. 2012;141(1):9-32. doi: 10.1016/j.jep.2012.02.042.

17. Patwardhan B, Vaidya ADB. Natural products drug discovery: accelerating the clinical candidate development using reverse pharmacology approaches. Indian J Exp Biol. 2010 [citado el 2 de abril del 2019]; 48(3):220-7. Disponible en: http://nopr.niscair.res.in/ handle/123456789/7395.

18. The World Medical Association. Declaración de la ANM sobre las consideraciones éticas de las bases de datos de salud y los biobancos. Declaración de Taipei. [Internet]. 2016 [citado el 2 de abril del 2019]. Disponible en: https://www.wma.net/es/policies-post/declaracionde-la-amm-sobre-las-consideraciones-eticas-de-las-bases-de-datosde-salud-y-los-biobancos/.

19. Elizagaray Fernández B, Castro Armas R. Producción científica cubana sobre plantas medicinales y productos naturales a partir de la base de datos PlantMedCUBA, 1967-2010. Rev Cubana Plant Med 2013; [citado el 11 diciembre del 2019] 18(3):348-60. Disponible en: http:// scielo.sld.cu/scielo.php?pid=S1028-47962013000300003\&script $=$ - ci_arttext\&tlng=pt.

20. Córdova Rengifo J. Uso y utilización de plantas medicinales en universidades de Lima [tesis para optar el título de licenciado en Antropología]. Lima: Pontificia Universidad Católica del Perú; 2011 [citado el 8 de enero 2020]. Disponible en: http://tesis.pucp.edu.pe/ repositorio/handle/20.500.12404/1077.

21. Organización Panamericana de la Salud. Situación de las plantas medicinales en Perú. Informe de reunión del grupo de expertos en plantas medicinales. [Internet]. OPS; [citado el 11 diciembre del 2019]; 2018. Disponible en: https://iris.paho.org/handle/10665.2/50479. 
22. Tilburt JC, Kaptchuk TJ. Herbal medicine research and global health: an ethical analysis. Bull World Health Organ. 2008;86(8):594-9. doi: 10.2471/BLT.07.042820.

23. Batugal P, Kanniah J, Lee S, Oliver J. Medicinal Plants Research in Asia - Volume I: The Framework and Project Workplans. Malasia: Bioversity International; 2004.

24. Kawamura M, Thomas CD, Tsurumoto A, Sasahara H, Kawaguchi Y. Lotka's law and productivity index of authors in a scientific journal. J Oral Sci. 2000;42(2):75-8. doi: 10.2334/josnusd.42.75.

25. International Society for Ethnopharmacology. Journal of Ethnopharmacology [Internet]. Elsevier; 2019 [citado 10 enero 2020]. Disponible: https://www.journals.elsevier.com/journal-of-ethnopharmacology.
26. Benedetti V, Echeverria G, Riquelme I. Biomedical research in Latin America: we can do more. The Lancet. 2016;387(10022):941. doi: 10.1016/S0140-6736(16)00567-5.

27. Ciocca DR, Delgado G. The reality of scientific research in Latin America; an insider's perspective. Cell Stress and Chaperones. 2017;22(6):847-52. doi: 10.1007/s12192-017-0815-8 .

28. Wagner CS, Park HW, LeydesdorffL. The Continuing Growth of Global Cooperation Networks in Research: A Conundrum for National Governments. PLOS ONE. 2015;10(7):e0131816. doi: 10.1371/journal.pone.0131816.

29. Alonso JAG, Saa MJM, Yugcha J del PH, Pilataxi RCM. Impacto de las publicaciones sobre plantas medicinales reportadas en Scopus. Rev Cubana Plant Med. 2018; [citado 10 enero 2020]. 22(3). Disponible en: http://www. revplantasmedicinales.sld.cu/index.php/pla/article/view/695. 